\title{
Los efectos de políticas públicas incoherentes. La relación entre los congresos médicos y el uso discrecional de la cesárea en México
}

\section{The effects of incoherent public policies. The relationship between medical congresses and the discretionary use of the cesarean section in Mexico}

\author{
Ana Jeanette Rojas Cruz \\ Centro de Investigación y Docencia Económicas. Región Centro (México) \\ rojascruzana@gmail.com
}

\section{NOTA BIOGRÁFICA}

Investigadora asistente en materia de políticas públicas. Actualmente se desempeña en materia de investigación y análisis de corrupción en gobiernos subnacionales en el estado de Aguascalientes, México.

Oliver David Meza Canales

Centro de Investigación y Docencia Económicas. Región Centro (México) ORCID: https://orcid.org/0000-0003-0342-3423

\section{NOTA BIOGRÁFICA}

Doctor en políticas públicas y actualmente investigador de tiempo completo en materia de políticas públicas, con especialidad en gobiernos locales y administración pública subnacional.

\section{RESUMEN}

En esta investigación discutimos la importancia que tiene poner énfasis en las políticas públicas que son incoherentes entre sí, en materia de eficacia del Estado. Utilizamos el caso de la práctica de la cesárea en México para entender cómo el fenómeno puede parcialmente explicarse a partir de la incoherencia entre instrumentos de la política de salud del gobierno mexicano. Preguntamos ¿hasta qué punto la realización de congresos médicos de ginecología y obstetricia, como parte de un proceso de certificación médica, se relaciona con la práctica de la cesárea en México, en el marco de una política pública que intenta acotar el uso discrecional de este método? Sostenemos que los objetivos de una política se ven afectadas por los instrumentos de otra política, y que por tanto el uso discrecional de este método de nacimiento está asociado por la incoherencia experimentada a partir de la presencia física que exige la certificación mediante congresos. Para demostrarlo se calculó la tasa de cesáreas por día de los años 2008 a 2015, y rastreamos las fechas de los congresos nacionales de ginecología y obstetricia para el mismo periodo de tiempo. Determinamos la asociación estadística entre ambos sucesos a partir de un análisis de regresión múltiple. El resultado es que existe una asociación estadística entre la tasa y la existencia de los congresos soportando el argumento de la incoherencia. En las conclusiones se proponen algunas ideas de política para mitigar este problema en lo particular, y en general en atender la incoherencia entre políticas del Estado.

\section{PALABRAS CLAVE}

Políticas públicas; coherencia de política; diseño de políticas; práctica de cesáreas; regresión múltiple. 


\begin{abstract}
In this research we discuss the importance of putting emphasis on public policies that are incoherent with each other, in terms of State effectiveness. We use the case of the practice of caesarean section in Mexico to understand how the phenomenon can be partially explained from incoherence between instruments of the Mexican government's health policy. We asked to what extent the holding of gynecology and obstetrics medical congresses, as part of a certification process, is related to the practice of caesarean sections, within the framework of a policy that attempts to limit the discretionary use of caesarean section in Mexico? We argue that the objectives of one policy are affected by the instruments of another policy, and that therefore the discretionary use of this birth method is associated with the incoherence experienced from the physical presence that requires certification through congresses. To demonstrate this, we calculated the rate of caesarean sections per day from 2008 to 2015, and we tracked the dates of the national gynecology and obstetrics congresses for the same period of time. We determine the statistical association between both events from a multiple regression analysis. The result is that there is a statistical association between the rate and the existence of congresses, supporting the argument of incoherence. The conclusions propose some policy ideas to mitigate this problem in particular, and in general to address the inconsistency between policies.
\end{abstract}

\title{
KEYWORDS
}

Public policy; policy coherence; policy design; cesarean section; multiple regression.

\section{SUMARIO}

INTRODUCCIÓN. 1. COHERENCIA ENTRE POLÍTICAS PÚBLICAS. 1.1. COHERENCIA ENTRE LOS INSTRUMENTOS DE POLÍTICA PÚBLICA. 2. LA PRÁCTICA DE LA CESÁREA. 2.1. LOS CONGRESOS MÉDICOS COMO FACTOR QUE AFECTA EL USO DE LA CESÁREA. 3. METODOLOGÍA. 3.1. DATOS.

3.2. VARIABLES. 3.2.1. Variable dependiente. 3.2.2. Variables independientes. 3.2.3. Variables control. 3.3. MODELO. 4. RESULTADOS. 5. DISCUSIÓN. 6. CONCLUSIONES. ANEXO 1. REFERENCIAS BIBLIOGRÁFICAS.

\section{INTRODUCCIÓN}

Los problemas públicos son complejos y multidimensionales (Meza, et al., 2018; Crowley y Head, 2017; Newman y Head, 2017). Existen políticas públicas que al ser analizadas individualmente son coherentes y alcanzan sus objetivos. Sin embargo, al analizarlas en conjunto con otro tipo de políticas, son incoherentes y pierden toda capacidad de sinergia, lo cual genera efectos negativos en otras políticas o problemas públicos (Cejudo y Michel, 2016; Howlett y Rayner, 2007). En este estudio ponemos énfasis en analizar los resultados de políticas públicas que mantienen incoherencia entre sí, para ello discutimos la importancia que tiene la coherencia externa en materia de eficacia del Estado. Aspecto que, a diferencia de los estudios sobre coherencia interna, la coherencia externa ha sido menos estudiada (Meza, 2018).

En este artículo utilizamos el ejemplo de las cesáreas en México para analizar cómo este fenómeno está parcialmente afectado por la incoherencia entre instrumentos de políticas públicas, ambos pertenecientes a la política de salud del Estado Mexicano. El uso de la cesárea se ha propagado, para algunos, como una epidemia que afecta a las mujeres que son madres. Tan solo en México, en 2018, 46,1\% de los nacimientos sucedieron bajo esta modalidad (ENADID, 2018) mientras que en América Latina el porcentaje fue de 40,5\% (Beltrán, et al., 2016).

La literatura que explica este fenómeno observa factores relacionados directamente a la oferta y demanda de los servicios de salud. Esta investigación toma cierta distancia de estos factores trayendo a la luz la falta de coherencia de instrumentos de política pública como un factor relevante en la práctica de la cesárea. En este caso específico, nos referimos al Programa de Acción Específico Salud de Materna y Perinatal (2013-2018) del Sistema Nacional de Salud y la certificación de los médicos especialistas de Ginecología y Obstetricia del Comité Normativo Nacional de Consejos de Especialidades Médicas (CONACEM) y los Consejos Médicos de Especialidades.

La pregunta de investigación que guía el estudio es ¿hasta qué punto la realización de congresos médicos de ginecología y obstetricia, como parte de un proceso de certificación médica, se relaciona con la práctica de la cesárea en México, en el marco de una política pública que intenta acotar el uso discrecional 
GAPP. Nueva Época - N. 24, noviembre 2020 - ISSN: 1989-8991 - DOI: https://doi.org/10.24965/gapp.i24.10792 - [Págs. 24-43]

Los efectos de políticas públicas incoherentes. La relación entre los congresos médicos y el uso discrecional de la cesárea en México

de este método? El argumento sostiene que parte del problema en el uso discrecional de la práctica de la cesárea en México se puede apreciar ante la falta de coherencia existente entre instrumentos de política pública, en este caso, la asistencia a congresos médicos de ginecología y obstetricia.

Por un lado, se encuentran las acciones del gobierno mexicano al reconocer que se tiene una alta tasas de cesáreas a nivel mundial; y por otro lado las acciones del gobierno de mantener capacitados y actualizados a los médicos ginecólogos y obstetras. La paradoja consiste en que dos instrumentos diseñados y promovidos por el Estado Mexicano para mejorar la calidad de los servicios de atención en salud entran en contradicción pues la implementación de un instrumento está asociado con decisiones sobre la práctica de la cesárea de manera innecesaria.

Para demostrar lo anterior, analizamos el comportamiento de la tasa de cesáreas (por día) antes, durante y después de la realización de los congresos médicos. La hipótesis que probamos es que (H1) durante los días previos de los congresos la tasa de cesáreas aumenta. El supuesto es que se programan anticipadamente nacimientos por cesárea para evitar que sucedan durante las fechas del congreso, que pueda comprometer la posibilidad de asistir al evento. Posteriormente discutimos estos resultados.

El artículo se organiza de la siguiente manera. En el primer apartado analizamos el marco de coherencia entre políticas públicas y en instrumentos de política pública. En el segundo apartado, contextualizamos el problema de la práctica de la cesárea en México y de la realización de los congresos médicos en México, y su posible relación con el uso discrecional de la cesárea. En el tercer apartado describimos la metodología empleada para encontrar la relación y validar la hipótesis. Después, en el apartado resultados que mostramos que existe una relación significativa entre la realización de congresos médicos y el uso de la cesárea como método de nacimiento. En el cuarto apartado se presenta una breve discusión de los resultados y la teoría, y también se presentan algunas consideraciones del estudio. Por último, como parte de las conclusiones presentamos tres propuestas de política pública, una en lo particular sobre la práctica de la cesárea; y en lo general dos propuestas sobre la importancia del análisis de coherencia externa en el diseño de las políticas públicas.

\section{COHERENCIA ENTRE POLÍTICAS PÚBLICAS}

En el estudio de las políticas públicas existen dos tipos de coherencia: la coherencia interna se refiere a la teoría causal que dio origen a la política pública (Meza, 2018; Cejudo y Michel, 2016) y a la coordinación que debe existir entre procesos y estructuras (Christensen y Laegreid, 2008; Peters, 2004; Pollitt, 2003). La coherencia externa, es el proceso mediante el cual los gobiernos hacen el esfuerzo de diseñar políticas que tomen en cuenta a otras políticas (Blouin, 2007) dado el supuesto de que comparten un conjunto de ideas y objetivos comunes (May et al., 2006).

Bajo la premisa de que los problemas públicos son complejos y multidimensionales (Meza, et al., 2018; Crowley y Head, 2017; Newman y Head, 2017; Head y Alford, 2013; Rittel y Webber, 1973), la coherencia externa entre políticas públicas existe cuando los distintos instrumentos de política, cada uno de manera diferente, atienden y aportan una solución al mismo problema público. Sin embargo, la especificidad que caracteriza la elaboración de las políticas públicas puede traer consigo la falta de coherencia entre los instrumentos que la conforman (Cejudo y Michel, 2016; 2017). En este sentido, el potencial de una política reside en la contribución que su implementación hace a la combinación de políticas existentes independientemente si están encaminadas a resolver el mismo problema público o solo comparten objetivos generales (Howlett y Rayner, 2007).

El foco de análisis en este nivel de coherencia reside en la unión, complementariedad y sinergia de los instrumentos de política; a fin de garantizar transversalidad de las acciones, y minimizar la desconexión y falta de coherencia entre los programas de una política pública (Aguilar, 2009).

\subsection{Coherencia entre los instrumentos de política pública}

El análisis de coherencia entre políticas públicas puede ser de tres tipos: 1) coherencia entre los instrumentos de política pública, 2) coherencia entre la población objetivo de cada política pública y 3) la coherencia entre los objetivos de las políticas públicas (Cejudo y Michel, 2016).

Para este estudio, únicamente tomaremos en cuenta la coherencia entre los instrumentos de política pública, los cuales son las acciones que los gobiernos tienen y proponen, para lograr objetivos específicos, 
GAPP. Nueva Época - N. 24, noviembre 2020 - ISSN: 1989-8991 - DOI: https://doi.org/10.24965/gapp.i24.10792 - [Págs. 24-43]

Los efectos de políticas públicas incoherentes. La relación entre los congresos médicos y el uso discrecional de la cesárea en México

y que, éstos aportan al alcance de objetivos generales (Aguilar, 2009: 6). La falta de coherencia entre los instrumentos de política pública puede atribuirse a la cantidad de entidades involucradas en la solución del problema, aunque en teoría, los distintos instrumentos que componen las políticas públicas deberían corresponderse por el hecho de que comparten un conjunto de ideas y objetivos (May et al., 2006). Sin embargo, un mayor número de actores involucrados implica una alta transferencia de información y altos grados de coordinación; y por lo general, no existen capacidades institucionales suficientes (Peters, 1998). Deficiencia que los gobiernos han tratado de resolver con la creación de más estructuras y procesos articulen las reglas para una mejor toma de decisiones (Cejudo y Michel, 2015).

Entonces, ¿en dónde son tomadas las decisiones que fomentan o perjudican la coherencia entre los instrumentos de las políticas públicas? para responder a esta pregunta, es preciso mencionar las barreras institucionales como factores que inciden en la coherencia entre los instrumentos de política pública. De acuerdo con Stead (2008) la primera barrera institucional que se debe considerar, y la más importante para fines de este estudio, tiene que ver con el desarrollo de políticas o programas de manera aislada. La cual, genera un bajo nivel de coherencia, tanto en la rendición de cuentas como el cálculo de los efectos de la política; y esto sucede porque se trata de acciones que se realizan dentro de la institución que busca resolver el problema público desde su ámbito. La segunda barrera tiene que ver con las redes formales y la influencia que hay entre los distintos actores involucrados en las políticas de diversos espacios de política.

La falta de coherencia entre estos instrumentos es evitable mediante la consideración de medidas apropiadas que mitiguen los impactos que una política pueda generar sobre otra, con el fin de evitar efectos neutralizadores (Blouin, 2007).

\section{LA PRÁCTICA DE LA CESÁREA}

La cesárea, como procedimiento quirúrgico, es considerada uno de los avances más importantes de la medicina perinatal contemporánea por el impacto que ha tenido en la disminución de la morbimortalidad materna y perinatal (OMS, 2015; Secretaría de Salud, 2002). El problema surge cuando su práctica se realiza discrecionalmente y responde a intereses ajenos a razones médicas y al bienestar humano (González-Pérez et al., 2011) sin tomar en cuenta los riesgos asociados al procedimiento (Secretaría de Salud, 2002).

En 1985, la Organización Mundial de la Salud (OMS) estimó que la tasa de cesáreas debería oscilar entre el $10 \%$ y $15 \%$ del total de partos; porque se evidenció que, si la tasa de cesáreas oscila en ese rango, tiene un impacto en la disminución del número de defunciones maternas y de recién nacidos. Sin embargo, cuando va por encima de este rango, no hay indicios de que mejoren las tasas de mortalidad (OMS, 2015).

La práctica de la cesárea, a nivel poblacional puede tener efectos para la madre y el recién nacido. Los efectos de la práctica de cesáreas en mujeres están asociados a un mayor riesgo de ruptura uterina, inserción anormal de la placenta, embarazos ectópicos, muerte fetal, parto prematuro; los cuales aumentan ante una mayor exposición al procedimiento (Sandall, et al. 2018). En los recién nacidos, los efectos de nacer mediante cesárea tienen que ver con una exposición hormonal, física, bacteriana y médica que pueden alterar la fisiología neonatal. Y tienen relación con padecimientos como alergias, asma y alteraciones en el microbiota intestinal. Los efectos a largo plazo aún están en investigación. Algunos estudios encuentran una relación positiva entre el nacimiento mediante cesárea y una mayor incidencia de asma y obesidad durante la niñez (Sandall, et al. 2018).

En un estudio realizado por Beltrán et al. (2016) en México, sobre la tendencia de la tasa de cesáreas a través de los años, muestran que en 2014 el $18,6 \%$ del total de nacimientos a nivel mundial fueron a través de cesáreas con una variación de entre $6 \%$ y $27 \%$ entre países con diferentes niveles de desarrollo. En América Latina y el Caribe, en promedio, el $40,5 \%$ de los nacimientos son a través de cesáreas, lo que la ubica a la región con la mayor tasa de cesáreas a nivel mundial, seguido por Norteamérica con una tasa de cesáreas de 32,3\%. Según la información de la Encuesta Nacional de la Dinámica Demográfica (ENADID) 2018, en México el 46,1\% de los partos atendidos fueron por cesárea, lo que ubica al país por encima del promedio mundial, y muy por encima de la recomendación que emite la OMS. Del total, $23,3 \%$ de los partos fueron por cesáreas programadas, y los estados con mayor porcentaje de cesáreas programada fueron Tamaulipas (33,3\%), Nuevo León (31\%) y Ciudad de México $(29,1 \%)$.

La preferencia por las cesáreas, en detrimento del parto natural, es multicausal. Por un lado, están los factores asociados al estado de salud de la mujer y el producto (Bernal-García y Nahín-Escobedo, 2018). Por otro lado, están los factores asociados a la prestación de los servicios de salud; los médicos y los hos- 
GAPP. Nueva Época - N. 24, noviembre 2020 - ISSN: 1989-8991 - DOI: https://doi.org/10.24965/gapp.i24.10792 - [Págs. 24-43]

Los efectos de políticas públicas incoherentes. La relación entre los congresos médicos y el uso discrecional de la cesárea en México

pitales (Bernal-García y Nahín-Escobedo, 2018; González-Pérez et al., 2011). Sin embargo, la actitud del médico ante el procedimiento de la cesárea juega un papel fundamental que determina la predisposición de las mujeres sobre la elección de la cesárea como método de nacimiento (Vallejos-Parás et al., 2011). La frecuencia de cesáreas cada vez es mayor y atiende a indicaciones que parecen no justificarse, por ejemplo, la facilidad de la cirugía a la vigilancia (en muchas ocasiones prolongada) del trabajo de parto (Muñoz-Enciso et al. 2011).

Algunos estudios (Burns, 1995; Hurst y Summey 1984) encuentran una relación positiva entre la especialización médica y el parto por cesárea. Por ejemplo, si la atención prenatal ha sido brindada por un especialista (obstetra) aumenta la probabilidad de que el nacimiento ocurra por cesárea. De acuerdo con Tussing y Wojtowicz (1993) también existe una relación positiva entre la categoría académica del médico y el parto por cesárea; si un residente es quien atiende el parto es más probable que sea por cesárea, de tal manera que los hospitales donde se realizan residencias médicas de obstetricia tienen mayores tasas de cesárea. Por otra parte, cuestiones relativas al tiempo juegan un papel importante. En el estado de Colima en México, un estudio encuentra que los patrones en la práctica de cesáreas estan asociados con factores como días de la semana y los horarios (González et al., 2011). En los hospitales del sector público es más económico realizar partos naturales que una cesárea, pero en cuestión de rapidez y eficiencia la cesárea trae beneficios pues como señala Karen Luna ${ }^{1}$ «muchas veces no hay camas, ni médicos suficientes» (The New York Times, 2017).

El Estado mexicano, en atención al problema de las cesareas en México, ha determinado una serie de acciones en este aspecto. En este caso, el Programa de Acción Específico Salud Materna y Perinatal (2013-2018) del Sistema Nacional de Salud representa la política pública en materia de salud materna y perinatal porque instrumentaliza, a través de estrategias y líneas de acción, las propuestas institucionales y de coordinación en el tema de salud materna y perinatal. El objetivo general del PEA es mejorar la calidad de los servicios de salud, su efectividad, el seguimiento y la rendición de cuentas para disminuir los rezagos de salud que afectan a la población.

De acuerdo con la práctica de la cesárea innecesaria, en este programa en el diagnóstico y la descripción de la problemática se menciona lo siguiente: «en el Sistema de Salud en algunas instituciones se ha observado un incremento por arriba de lo normado [...]. En el 2012 se identificaron instituciones que tenían más del $50 \%$ de la atención de sus nacimientos por cesáreas» (pág. 35). Entre las prioridades del programa se encuentran: garantizar acceso universal, mejorar la calidad de la atención, contar con recursos humanos calificados y generar información estratégica. La reducción de cesáreas innecesarias se instrumentaliza en la prioridad «Mejorar la calidad de la atención» mediante el cumplimiento del Lineamiento Técnico sobre indicación y práctica de la operación cesárea de la Dirección General de Salud Reproductiva de la SSA.

En atención a la problemática, en el Programa, dentro de los objetivos se encuentra el Objetivo Específico 1 «Propiciar el acceso universal de mujeres en etapas pregestacional, embarazo, parto, puerperio y neonatal, a servicios de calidad y respetuosos». El cual, delinea la estrategia 1.1. Incrementar el acceso en las etapas pregestacional, prenatal, parto, puerperio y neonatal a servicios con alta calidad; la cual contiene la línea de acción 1.1.1. Contribuir a mejorar la calidad y seguridad en la atención, con base en evidencia científica y mejores prácticas, eliminando intervenciones nocivas e innecesarias.

\subsection{Los congresos médicos como factor que afecta el uso de la cesárea}

La realización de congresos médicos es parte de las acciones que hace el Estado en reconocimiento de la existencia de un problema público, en este caso, de asimetría de información y atención a la regulación del ejercicio de la medicina. En muchos países los gobiernos incorporan políticas públicas para asegurar que los especialistas médicos cuenten con certificación y recertificaciones profesionales para mantener actualizado el conocimiento en sus ramos, y para asegurar que quien brinde servicios de este tipo, tenga el perfil apropiado. En el caso mexicano, este proceso de calidad pasa por la asistencia a congresos médicos. Explicamos brevemente.

Anteriormente la certificación de especialidades médicas no era obligatoria, bastaba con la obtención del título de especialidad para ejercer; de tal manera que cualquier médico general tenía la posibilidad de ejercer actividades como especialista con el solo hecho de cursar diplomados o maestrías sobre algún tema

1 Investigadora jurídica del Grupo de Información en Reproducción Elegida. 
GAPP. Nueva Época - N. 24, noviembre 2020 - ISSN: 1989-8991 - DOI: https://doi.org/10.24965/gapp.i24.10792 - [Págs. 24-43]

Los efectos de políticas públicas incoherentes. La relación entre los congresos médicos y el uso discrecional de la cesárea en México Ana Jeanette Rojas Cruz / Oliver David Meza Canales

relacionado a una especialidad médica; sin contar con la práctica y conocimiento que se adquieren durante la residencia médica.

En el caso de la especialidad médica de ginecología y obstetricia, la certificación tiene el objetivo de demostrar que el especialista cuenta y mantiene la competencia para el ejercicio profesional; la obtención de la certificación analiza y registra la actualización y aprendizaje personal del médico especialista por medio de distintas actividades.

En México, la certificación médica se hizo obligatoria desde el 1 de septiembre de 2011 a través de modificaciones a la Ley General de Salud, específicamente al artículo 272 Bis. El artículo señala:

«para la realización de cualquier procedimiento quirúrgico de especialidad, los profesionales que lo ejerzan además de contar con una cédula que acredite la especialidad, deben contar con un certificado vigente de especialista que acredite la capacidad y la experiencia en la práctica de los procedimientos y técnicas correspondientes en la materia. El cual, deberá ser expedido por el Consejo de la especialidad, estipulado en el artículo 81 de la misma Ley.»

El organismo encargado de expedir los certificados médicos a especialistas en ginecología y obstetricia, así como sus derivados, es el Consejo Mexicano de Ginecología y Obstetricia (CMGO) que cuenta con la declaratoria de idoneidad del Comité Normativo Nacional de Consejos de Especialidades Médicas (CONACEM). El cual, tiene el objetivo de vigilar que los Consejos Médicos cumplan con la responsabilidad de discernir quiénes son los especialistas que se han preparado formalmente y cuentan con las facultades y conocimientos necesarios y actualizados para ejercer su profesión.

El proceso de certificación de ésta especialidad consiste en tramitar la certificación y revalidar su vigencia periódicamente. La recertificación puede llevarse a cabo de dos maneras: por puntaje y por realización de examen de vigencia. Durante el primer año de caducidad de la vigencia se requiere un total de 250 puntos para la recertificación, en el segundo año 300 puntos y al tercer año 350. Únicamente es tomado en cuenta el puntaje obtenido en los últimos cinco años. El esquema de incentivos hace que los médicos prefieran mantener la certificación por puntaje, ya que el costo de rechazar esta opción es más alto porque las certificaciones posteriores tendrían que ser por examen. Para la certificación por puntaje, el CMGO cuenta con un listado del tipo de actividades, y el número de puntos que otorga cada uno (ver Tabla 1).

Tabla 1. Congresos en Ginecología y Obstetricia. Tipos, actividades y puntaje que otorgan

\begin{tabular}{ll}
\hline Congreso & Puntaje \\
\hline Nacional & 4 tipos de actividades que van de los 6 a 18 puntos por cada una \\
\hline Regional & 4 tipos de actividades que van de los 6 a 12 puntos por cada una \\
\hline Estatal & 6 tipos de actividades que van de los 4 a 10 puntos por cada una \\
\hline Mundial & 4 tipos de actividades que van de los 4 a 12 puntos por cada una \\
\hline
\end{tabular}

Fuente: Elaboración propia con datos de la CMGO sobre la Cédula de Vigencia de Certificación.

Para que sea tomado en cuenta el puntaje, cada médico debe presentar la documentación que avale la actividad. El Comité de Vigencia de la CMGO será el encargado de validar que la información presentada sea veraz y completa.

Los congresos son importantes para la vida profesional de los médicos. Con ellos, el personal se actualiza en temas de interés, socializa con pares, conoce destacados investigadores o autores, conoce pares interesados en los mismos temas o líneas de investigación, e intercambia ideas o establecer vínculos de trabajo, entre otros (Elizalde, 2017). En el ámbito de la medicina ginecológica y obstétrica, los congresos son actividades coordinadas y organizadas por la Federación Mexicana de Colegios de Obstetricia y Ginecología (FEMECOG). Está compuesta por 75 colegios y asociaciones, distribuidas en siete regiones en el país y cuenta con poco más de cinco mil miembros. Los congresos nacionales son abiertos a todos los médicos de la especialidad y subespecialidades del país, y los congresos regionales están dirigidos directamente a los interesados de cada estado componente de la región. Por lo general, los congresos tanto nacionales como regionales, tienen una periodicidad anual y el lugar (estado y municipio) de realización cambia. La certifica- 
ción, y sobre todo su vigencia, depende en gran medida de la asistencia y participación en los congresos. De tal manera que, para mantener una certificación, existe un gran incentivo para asistir a los congresos médicos de la especialidad.

En resumen, la certificación médica de los especialistas en ginecología y obstetricia soluciona problemas de asimetría de información y regulación por lo que de esta forma la CONACEM y los CMGO coadyuvan con el objetivo del Estado de proteger el derecho a la salud de las personas (Wolpert, 2017). La certificación funge como auxiliar para las autoridades gubernamentales de tener mayor certeza sobre la profesionalización de los médicos que llevan a cabo procedimientos quirúrgicos de especialidad, así como de su capacidad y pericia (Wolpert, 2017). La pregunta es si esta certificación tiene relación directa con el uso discrecional de la cesárea y por tanto de exponer a las mujeres a mayores riesgos de los que son necesarios por interés diferente al bienestar de ellas, que es algo que intenta ser acotado a partir de las lineas de política que señala el Programa de Acción Específica de Salud Materna y Perinatal 2013-2018.

La siguiente sección se encarga de la parte empírica donde la hipótesis se pone a prueba. Como parte de los factores que explican el incremento de las cesáreas en México, aquí se argumenta que parte del problema de la práctica de la cesárea puede explicarse por la falta de coherencia entre instrumentos de política pública. Por tanto, se señala que los congresos para los especialistas en ginecología y obstetricia son motivo para el uso discrecional de la programación de cesáreas. La expectativa empírica es que durante los días previos de los congresos la tasa de cesáreas aumenta; bajo el supuesto de que los médicos programan anticipadamente nacimientos por cesárea para evitar que sucedan durante las fechas del congreso.

\section{METOdOLOGÍA}

El objetivo de este estudio es verificar la relación entre el uso discrecional de la cesárea y la realización de los congresos médicos de ginecología y obstetricia. En términos teóricos, si encontramos esta relación, estaríamos hablando de dos cursos de acción que el Estado dispone pero que el éxito de uno de los cursos tiene efectos inesperados en el otro curso de acción. Para poder responder la pregunta de investigación y poner a prueba la hipótesis y el argumento que guían el estudio, realizamos un proceso metodológico que comprende: los datos, en donde justificamos a detalle la recolección de la información; las variables, en donde explicamos la cómo se integró cada variable y su relevancia; el método, donde justificamos la elección del método de mínimos cuadrados ordinarios para estimar los parámetros del modelo de regresión múltiple.

\subsection{Datos}

Los datos utilizados para realizar este análisis corresponden a los nacimientos por cesárea y congresos nacionales para médicos especialistas en ginecología y obstetricia en México. Los datos provienen de dos fuentes: el número de nacimientos por cesárea se obtuvo de la base de datos de Certificados de Nacimiento $(\mathrm{CdN})$ de la Secretaría de Salud (SSA) ${ }^{2}$. Las fechas de los congresos corresponden a los organizados anualmente a nivel nacional por la Federación Mexicana de Colegios de Obstetricia y Ginecología (FEMECOG) ${ }^{3}$.

En México hay dos fuentes de las que se puede extraer información sobre cesáreas. Una es la ENADID, del Instituto Nacional de Estadística y Geografía (INEGI) que provee información pública sobre el porcentaje de cesáreas. Sin embargo, por ser una encuesta especial, tiene un periodo de levantamiento variable por lo que no podrían hacerse inferencias adecuadas sobre el comportamiento de la tasa de cesáreas a través del tiempo.

Para este estudio optamos por los datos de los CdN para el cálculo de la tasa de cesáreas porque los certificados tienen información precisa sobre el tipo procedimiento (cesárea), el momento (día, mes, año y hora) y lugar (estado, municipio, localidad) de cada nacimiento.

En México se celebran distintos tipos de congresos: nacionales, regionales y estatales. Para este estudio tomamos en cuenta las fechas de los congresos médicos en Ginecología y Obstetricia de la FEMECOG porque la asistencia es abierta a todo médico especialista en Ginecología y Obstetricia y subespecialidades

\footnotetext{
2 La base es pública y puede ser consultada en la página de la Dirección General de Información en Salud en el apartado Conocimiento en Salud, subapartado Datos Abiertos y por último en la opción Nacimientos.

3 En el apartado referencias se encuentran las páginas de internet que fueron consultadas para rastrear las fechas de realización de cada congreso nacional del año 2008 al año 2015.
} 
GAPP. Nueva Época - N. 24, noviembre 2020 - ISSN: 1989-8991 - DOI: https://doi.org/10.24965/gapp.i24.10792 - [Págs. 24-43]

Los efectos de políticas públicas incoherentes. La relación entre los congresos médicos y el uso discrecional de la cesárea en México

relacionadas, mientras que, a los congresos regionales o estatales, sólo pueden acudir los médicos que corresponden a ese territorio. Otra razón por considerar fue la disponibilidad de la información; las fechas y lugares donde se han llevado a cabo los congresos regionales o estatales durante el periodo de tiempo de análisis no está disponible para todas las regiones.

\subsection{Variables}

Para correr el modelo de mínimos cuadrados ordinarios, integramos una base de datos de $\mathrm{N}=93,465$ observaciones. Esta base contiene información de la variable dependiente la tasa de cesáreas por día durante un periodo de tiempo del año 2008 al año 2015 para cada una de las entidades federativas de México. Las variables independientes que son los días previos, durante y después de cada congreso anual, y un vector de variables control sobre el año, día, mes, entidad y si la entidad fue o no sede del congreso. El vector tiene la intención de controlar todos los efectos de factores que no se contemplan dentro del modelo.

\subsubsection{Variable dependiente}

La variable dependiente es el promedio de la tasa de cesáreas; para la construcción de esta variable fue necesario obtener el número total de nacimientos mediante cesárea por día ocurridos entre el año 2008 y el año 2015 en cada una de las 32 entidades federativas, después con información poblacional de la ENADID sobre la cantidad de mujeres en edad reproductiva ( 15 a 49 años) para el mismo periodo de tiempo para cada entidad, calculamos el promedio de la tasa de cesáreas de la siguiente manera:

tasa de cesáreas $_{i}=\left(\#\right.$ de nacimientos por cesaria ${ }_{i} / \#$ mujeres en edad reproductiva $\left.{ }_{i j}\right){ }^{*} 100.000$ mujeres $^{4}$

\subsubsection{Variables independientes}

En los días previos al inicio del congreso, se espera que las tasas de cesáreas aumenten por lo que los médicos se ven en la necesidad de realizar los nacimientos con fecha probable de parto para las fechas del congreso, días antes, con la finalidad de evitar que se comprometa su asistencia al congreso, y también el riesgo de cualquier complicación de parto que no pueda ser atendida por personal capacitado. Para comprobar la hipótesis del estudio, integramos la variable días previos al congreso, la cual consiste en un vector de variables dicotómicas, en donde 1 identifica los cinco días previos de cada congreso anual. El valor de 0 identifica las observaciones de todos los demás días del año donde no hay congresos. En promedio en todos los años los congresos duran 5 días, por lo que tomó como referencia el mismo número de días antes del congreso.

Durante los días del congreso es de esperarse que la tasa de cesáreas disminuya porque no todos los médicos ejercen sus actividades normales durante este periodo de tiempo. Sin embargo, sería un error asumir que no ocurren cesáreas durante ese periodo de tiempo porque hay médicos que no asisten a los congresos y llevan a cabo sus labores normales. Por esta razón, el modelo contempla la variable días del congreso; que consiste en un vector de variables dicotómicas donde asignamos el valor de 1 a los días en que se llevaron a cabo los congresos y el valor de 0 a todos los demás días del año. En promedio, todos los congresos tienen una duración aproximada de 5 días.

En los días posteriores al congreso, lo esperado es que la tasa de cesáreas regrese a su comportamiento «normales». Para comprobarlo, elaboramos la variable días después del congreso; el cual consiste en un vector de variables dicotómicas donde asignamos el valor de 1 a los cinco días posteriores a la finalización de cada congreso anual; y el valor de 0 a los demás días del año. Al igual que los días previos al congreso, tomamos como referencia cinco días.

\subsubsection{Variables control}

Los controles para el estudio corresponden a variables que contienen información sobre el año al que corresponde la observación, los días de la semana, el mes y entidad federativa al que corresponde cada observación.

4 Donde $i$ corresponde a la fecha (día, mes y año) de interés. 
Incorporamos como variable control un vector de variables año con la finalidad de que esta variable capture algún evento o suceso que pueda afectar el comportamiento de la variable dependiente. La intención de agregar el vector de variables días de la semana corresponde a los hallazgos encontrados en el estudio de González, et al. (2011) sobre la práctica de la cesárea en los hospitales públicos del estado de Colima y en los hospitales públicos del país; entre los principales hallazgos del estudio se encuentra que la práctica de la cesárea aumenta los viernes, sábado y domingo; y en los turnos vespertinos y nocturnos. El vector de variables meses se incluyó como control porque los meses en que se llevan a cabo los congresos nacionales de la FEMECOG, por lo general, son de agosto a octubre. El vector de variables entidad para que capture las diferencias entre los estados y que esto no afecte el comportamiento de la variable dependiente. Por último, agregamos como variable control un identificador por año de la entidad sede del congreso, en donde el valor de 1 se asigna al estado sede y 0 a los demás estados participantes.

\subsection{Modelo}

Para responder a la pregunta ¿hasta qué punto la realización de congresos médicos de ginecología y obstetricia, como parte de un proceso de certificación médica, se relaciona con la práctica de la cesárea en México, en el marco de una política pública que intenta acotar el uso discrecional de este método? y probar la hipótesis de que durante los días previos a los congresos la tasa de cesáreas aumenta debido a que los médicos programan anticipadamente los nacimientos por cesárea para evitar que estos sucedan durante las fechas de los congresos y pueda comprometerse la asistencia al congreso; decidimos emplear un modelo de regresión múltiple con mínimos cuadrados ordinarios porque se trata de una prueba simple y la interpretación de sus resultados es directa y simple. Además, Wooldridge (2009), propone los análisis de regresión múltiple como los más útiles para explicar la variación de y en función de diversas variables explicativas cuando todo lo demás ceteris paribus porque permite controlar de manera explícita muchos otros factores que afectan simultáneamente a la variable dependiente.

El modelo sintético por emplear es el siguiente:

$Y i=\beta 0+\beta 1 \times 1+\beta 2 \times 2 \ldots+\beta k x k+u$.

Donde:

- $\beta 0$ es el intercepto.

- $\quad \beta 1$ mide el cambio en y respecto a $x 1$, manteniendo constantes todos los demás factores. En este caso y es la tasa de cesáreas para determinado día en determinado lugar.

- $\quad \beta 2$ mide el cambio en y respecto a $x 2$, manteniendo constantes todos los demás factores. La X2 relevante es en realidad un vector que identifica días antes, durante y después del congreso de ginecología y obstetricia.

- $\quad \beta k$ mide el cambio en y respecto a $x k$, manteniendo constantes todos los demás factores. Este otro vector son los controles para aislar la estimación relevante.

- $u$ término de error.

Con la sustitución de las variables que conforman el estudio, el modelo quedó de la siguiente manera:

- Tasa de cesáreas $=B 0+B 1$ días antes del congreso $+B 2$ días del congreso $+B 3$ días después del congreso $+B 4$ sede $+\angle 1$ año $+\angle 2$ día de la semana $+\angle 3$ mes $+\angle 4$ identidad federativa.

\section{RESULTADOS}

¿Qué relación existe entre la realización de los congresos médicos nacionales de Ginecología y Obstetricia, con el comportamiento de la tasa de cesáreas en México? La práctica de la cesárea es un problema público de salud que se ve afectado por la incoherencia entre dos políticas que coexisten en un mismo espacio y a pesar de que tienen objetivos particulares diferentes, durante su implementación generan un impacto en la práctica de la cesárea.

Los resultados muestran que controlando por día de la semana, mes, año y entidad federativa las variables relativas a los días previos al congreso médico, los coeficientes tienen dirección esperada y varios de ellos con magnitud estadísticamente significativa. Además, los datos están bien ajustados al modelo teórico al contar con una $\mathrm{r}^{2}$ de 0,864 . 
La gráfica 1 es útil para visualizar el impacto y la magnitud de los congresos en las tasas de cesáreas, pero los resultados estadísticos se muestran puntualmente en la tabla 2. Se analiza el movimiento en la tasa en tres grupos de datos; antes del congreso, durante el congreso y después del congreso. El grupo base para la comparación en esta estimación son los días que están fuera de las categorías antes mencionadas.

Los resultados son los siguientes. Primero, los días previos al congreso registran un incremento en la tasa de cesáreas. Estos coeficientes son estadísticamente significativos al $99 \%$ y $95 \%$ a excepción del quinto día antes del congreso; cinco días antes la tasa aumenta 0,0458 \% respecto a los demás días, sin embargo, la estimación no es significativa. El crecimiento en la tasa aumenta en el cuarto día previo al congreso (32,5\%), para el tercer día aumenta $18,9 \%$, para el segundo día $15,1 \%$ y un día antes $19,3 \%$.

Segundo, durante los días de congreso, el impacto es negativo; la tasa de cesáreas disminuye. Las estimaciones para este bloque de días son significativas al $99 \%$ y $95 \%$ a excepción del tercer día de congreso; y sugieren que el primer día de congreso la tasa de cesáreas disminuye $58 \%$, el segundo día disminuye $73,7 \%$, el cuarto día $6,8 \%$ y el quinto día $23,7 \%$ en comparación con los días que están fuera de estas categorías.

Tercero, las estimaciones relacionadas a los días posteriores al congreso muestran un patrón distinto a los días previos y los días que dura congreso. En este bloque de días hay impactos tanto positivos (aumento) como negativos (disminución) en la tasa de cesáreas. Sin embargo, el impacto de algunas estimaciones carece de significatividad estadística, por ejemplo, el primer y cuarto día. El segundo día posterior al congreso es significativo al $95 \%$ y la tasa de cesáreas aumenta en $14,2 \%$ respecto a los días fuera del bloque de análisis. El tercer día es estadísticamente significativo e implica un aumento del $25,7 \%$ en la tasa de cesáreas; el quinto día también es estadísticamente significativo e implica un aumento de $35,9 \%$ en la tasa de cesáreas. El comportamiento de la tasa de cesáreas en este bloque de días puede atribuirse a los demás factores relacionados con la tasa de cesáreas que quedan fuera del estudio, lo cual da soporte a la hipótesis y el argumento del estudio.

GRÁFICA 1. Estimadores díAs ANTES, DURANTE y DESPUÉS DEL CONGRESO

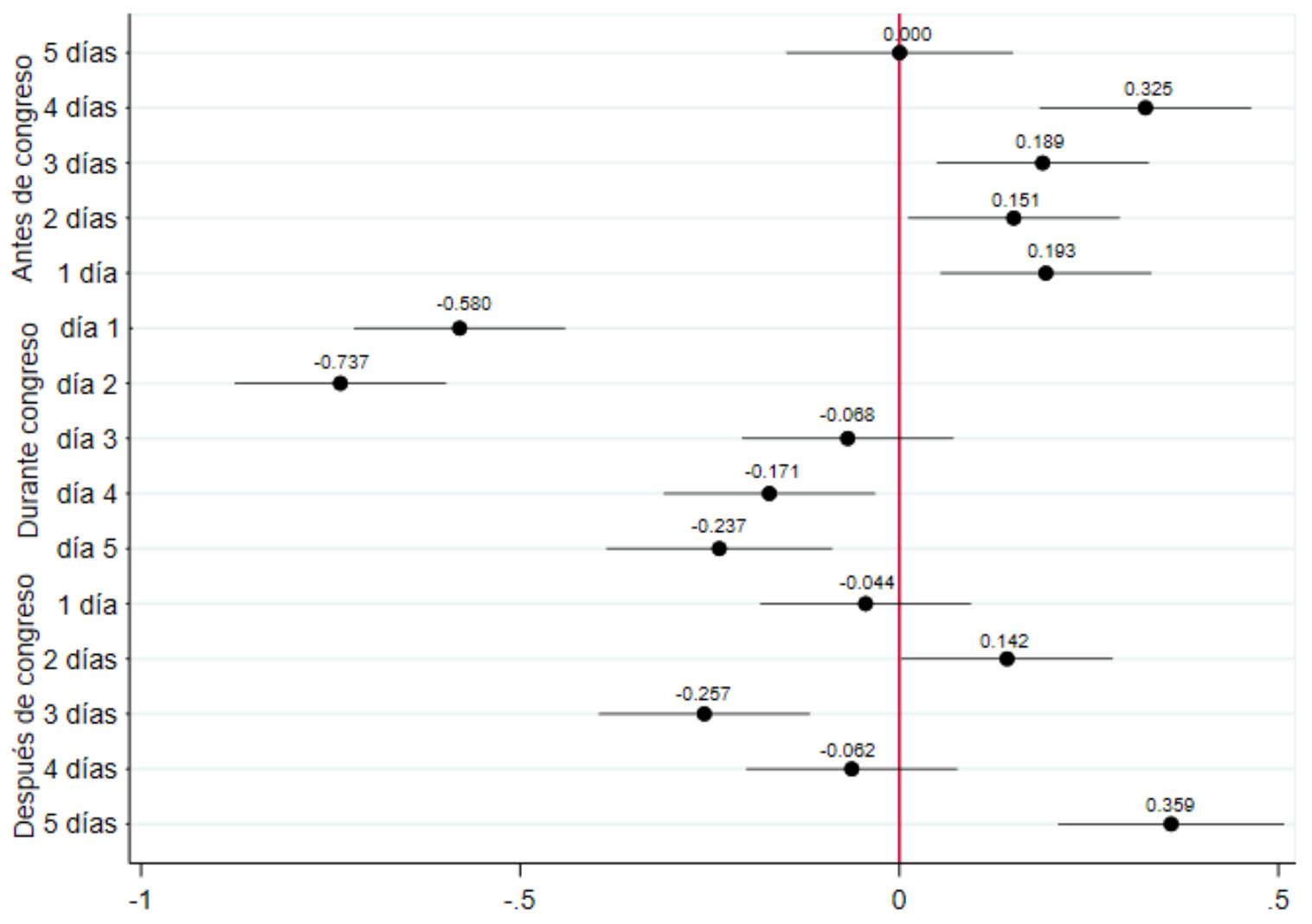

Fuente: Elaboración propia. 
GAPP. Nueva Época - N. 24, noviembre 2020 - ISSN: 1989-8991 - DOI: https://doi.org/10.24965/gapp.i24.10792 - [Págs. 24-43]

Los efectos de políticas públicas incoherentes. La relación entre los congresos médicos y el uso discrecional de la cesárea en México

En cuanto a la variable sede de congreso, la tasa de cesáreas aumenta $30,2 \%$ con nivel de significatividad estadística de $99 \%$.

Sobre las variables control año, todos son significativos al $99 \%$, en esta categoría el año base es 2008 . Los controles relacionados a los meses del año, al igual fueron significativos al 95 y 99 por ciento, enero es el mes base por lo que los estimadores de este vector tienen que interpretarse en comparación con enero. Algo que cabe resaltar es que los meses durante los que los congresos son llevados a cabo regularmente son agosto-noviembre, el impacto de los estimadores de estos mismos meses es más alto en la tasa de cesáreas en comparación con los otros meses, sin embargo, las estimaciones arriba ya son controladas por este hecho. Lo robustece el sustento sobre el cual tiene como base el argumento principal de la investigación, que los congresos médicos como mecanismo de capacitación y actualización del personal médico tiene un efecto aumentativo en la tasa de cesáreas.

En el vector días de la semana, todos son significativos al $99 \%$ y el día de comparación es el domingo, sin embargo, el patrón que aquí se encuentra con los estimadores es que el sábado es el día que tiene un menor impacto en la tasa de cesáreas en comparación con el domingo. El vector de entidades federativas se incorporó dentro de la ecuación de manera desglosada para identificar efectos de corte institucional o idiosincrático -nivel estado-. Sin embargo, se encuentra que todos los estados son significativos al 95 y 99 por ciento. En la tabla 2 se encuentran los valores estimados para cada una de las variables independientes y se especifica el uso de controles. En el apartado de Anexos se encuentra la tabla completa.

TABla 2. Especificaciones de REgresión POR Mínimos CuAdRados Ordinarios

\begin{tabular}{|c|c|c|}
\hline & Variables & Valores y grados de significancia \\
\hline \multirow{10}{*}{ Días antes del congreso } & \multirow[t]{2}{*}{5 días } & 0,000458 \\
\hline & & $(0,0763)$ \\
\hline & \multirow[t]{2}{*}{4 días } & $0,325^{\star * *}$ \\
\hline & & $(0,0713)$ \\
\hline & \multirow[t]{2}{*}{3 días } & $0,189^{* * *}$ \\
\hline & & $(0,0714)$ \\
\hline & \multirow[t]{2}{*}{2 días } & $0,151^{* *}$ \\
\hline & & $(0,0713)$ \\
\hline & \multirow[t]{2}{*}{1 día } & $0,193^{* * *}$ \\
\hline & & $(0,0711)$ \\
\hline \multirow{10}{*}{ Días de congreso } & \multirow[t]{2}{*}{1 día } & $-0,580^{* * *}$ \\
\hline & & $(0,0712)$ \\
\hline & \multirow[t]{2}{*}{2 día } & $-0,737^{* * *}$ \\
\hline & & $(0,0712)$ \\
\hline & \multirow[t]{2}{*}{3 día } & $-0,0680$ \\
\hline & & $(0,0712)$ \\
\hline & \multirow[t]{2}{*}{4 día } & $-0,171^{* *}$ \\
\hline & & $(0,0712)$ \\
\hline & \multirow[t]{2}{*}{5 día } & $-0,237^{* * *}$ \\
\hline & & $(0,0761)$ \\
\hline
\end{tabular}


GAPP. Nueva Época - N. 24, noviembre 2020 - ISSN: 1989-8991 - DOI: https://doi.org/10.24965/gapp.i24.10792 - [Págs. 24-43]

Los efectos de políticas públicas incoherentes. La relación entre los congresos médicos y el uso discrecional de la cesárea en México Ana Jeanette Rojas Cruz / Oliver David Meza Canales

\begin{tabular}{|c|c|c|}
\hline \multirow{10}{*}{ Después de congreso } & \multirow[t]{2}{*}{1 día } & $-0,0444$ \\
\hline & & $(0,0710)$ \\
\hline & \multirow[t]{2}{*}{2 día } & $0,142^{* *}$ \\
\hline & & $(0,0711)$ \\
\hline & \multirow[t]{2}{*}{3 día } & $-0,257^{* * *}$ \\
\hline & & $(0,0711)$ \\
\hline & \multirow[t]{2}{*}{4 día } & $-0,0627$ \\
\hline & & $(0,0711)$ \\
\hline & \multirow[t]{2}{*}{5 día } & $0,359^{* * *}$ \\
\hline & & $(0,0761)$ \\
\hline \multicolumn{2}{|c|}{$\begin{array}{c}\text { Sede congreso } \\
(0,0223)\end{array}$} & $0,302^{* * *}$ \\
\hline \multicolumn{2}{|c|}{ Años } & Sí \\
\hline \multicolumn{2}{|c|}{ Meses } & Sí \\
\hline \multicolumn{2}{|c|}{ Días de la semana } & Sí \\
\hline \multicolumn{2}{|c|}{ Entidades federativas } & Sí \\
\hline \multirow{2}{*}{\multicolumn{2}{|c|}{$\begin{array}{c}\text { Constante } \\
(0,0275)\end{array}$}} & $3,611^{\star * *}$ \\
\hline & & \\
\hline \multicolumn{2}{|l|}{$\mathrm{N}$} & 93,495 \\
\hline \multicolumn{2}{|c|}{ R-cuadrada } & 0,864 \\
\hline
\end{tabular}

Errores estándar entre paréntesis. Coeficientes significativos al ${ }^{* *} 1 \%,{ }^{* *} 5 \%,{ }^{*} 10 \%$. Categorías referencia entidad federativa Aguascalientes. Fuente: Elaboración propia.

\section{DISCUSIÓN}

Las políticas públicas son acciones del gobierno para dar solución a problemas públicos, sin embargo, en la práctica gran parte de estas acciones se contraponen y tienen resultados no esperados (Cejudo y Michel, 2016; Howlett y Rayner, 2007). Lo anterior, puede deberse a que durante el proceso de elaboración de una política pública gran parte del esfuerzo está encaminado en que la política sea armoniosa respecto a la teoría que le da origen (Meza, 2018), los procesos y las estructuras (Christensen y Laegreid, 2008; Peters, 2004; Pollitt, 2003); y se deja fuera del diseño el análisis que tome en cuenta la manera en que la política entrará y formará parte de un grupo de políticas (Cejudo y Michel, 2016).

Los instrumentos de evaluación de las políticas públicas más utilizados tienden a evaluar la lógica causal de una política pública, pero nada pueden hacer para garantizar la coherencia entre un grupo de políticas públicas (Cejudo y Michel, 2016). En la actualidad, no existen instrumentos que permitan identificar o lograr coherencia entre políticas públicas; existen mecanismos de gestión que pasan el análisis individual de una política, pero son insuficientes para observar la coherencia externa. Generalmente, se cae en el error de considerar que los mecanismos de planeación o alineación, coordinación, monitoreo y evaluación garantizan la idealidad de una política pública (Cejudo y Michel, 2016).

En este artículo se muestra como la práctica de la cesárea, independientemente de otras causas, es influenciada por la ejecución de otras acciones de política como los congresos médicos. Esta conclusión no tiene la intención de poner la atención sobre los médicos y las personas que recurren a esta práctica, en realidad se trata de una serie de incentivos sistémicos que les obliga o fuerza a realizar esta práctica. 
GAPP. Nueva Época - N. 24, noviembre 2020 - ISSN: 1989-8991 - DOI: https://doi.org/10.24965/gapp.i24.10792 - [Págs. 24-43]

Los efectos de políticas públicas incoherentes. La relación entre los congresos médicos y el uso discrecional de la cesárea en México

Los resultados anteriores sugieren que un factor importante en el estudio del comportamiento de la tasa de cesáreas son los congresos nacionales de Médicos en Ginecología y Obstetricia. En primer lugar, en los días previos a los congresos se encontró que parte del aumento de la tasa de cesáreas sí está relacionado con la asistencia de los médicos al congreso. Lo cual nos permite inferir que los médicos se ven «en la necesidad» de atender el mayor número de nacimientos posibles para que durante su ausencia no exista complicación alguna, aun cuando esto implica elegir el método de nacimiento que más se adecue a su agenda y no las necesidades de la paciente. Lo anterior, puede ser más evidente en los hospitales privados porque las mujeres pueden elegir el tipo de nacimiento que desea experimentar, y es vulnerable a ser más influenciable de que se realice una cesárea aun cuando esta no es necesaria.

Respecto a los días en los que se lleva a cabo el congreso, los resultados muestran que la tasa de cesáreas disminuye. Esto puede atribuirse a que las cesáreas no son única y exclusivamente practicadas por un médico especialista en ginecología y obstetricia; sino que también por los médicos residentes de esta especialidad, que, aunque aún no cuentan con licencia para ejercer este procedimiento cuentan con conocimientos suficientes para actuar en casos extraordinarios y de urgencia. Asimismo, también puede atribuirse a que no todos los ginecólogos y obstetras acuden anualmente a los congresos nacionales.

Resaltamos algunas limitantes de esta investigación. Al ceñir la investigación a un marco teórico de coherencia entre instrumentos de política pública, el estudio estuvo enfocado en encontrar una relación directa entre las variables de interés: la tasa de cesáreas y las fechas en que se realizan los congresos nacionales de ginecología y obstetricia. Tambien se dejan fuera factores importantes relacionados a la práctica de la cesárea. Otro aspecto relevante es que los resultados se verían afectados si el numero de nacimientos cambia de manera condicionada a las fechas del congreso. No tenemos una explicación teórica para sostener este supuesto. Sin embargo, dado que los congresos son celebrados en diferentes fechas, y el análisis se hizo con datos de varios años, este supuesto condicionado tendría, en el mejor de los casos, una contribución mitigada en los resultados obtenidos.

Respecto con la demanda del servicio, en futuras investigaciones sería interesante agregar variables sobre los aspectos económicos y sociales de las mujeres. Sobre la oferta de los servicios de salud, habría que considerar factores de calidad y cantidad de los servicios de salud, tanto públicos como privados; las características geográficas y demográficas de cada una de las entidades federativas, y las razones detrás de que los médicos especialistas en ginecología y obstetricia prefieran practicar una cesárea en lugar de un parto natural.

Lo anterior ayudaría a entender mejor el mecanismo del fenómeno develado, por ejemplo, las capacidades locales u hospitalarias podrían incrementar o disminuir el uso de cesárea aun cuando el especialista se encuentre fuera. En estudios posteriores pueden retomarse este tipo de variables explicativas en el modelo y de esta manera presentar resultados más robustos que puedan afirmar o revocar el argumento e hipótesis que se propusieron en esta investigación.

\section{CONCLUSIONES}

En este estudio demostramos la importancia que tiene atender la coherencia externa entre políticas públicas. Revisamos el caso de la práctica de la cesárea y la realización de los congresos de ginecología y obstetricia en México. Los hallazgos sugieren que dos instrumentos de política de salud en México se contraponen de forma que los resultados de uno afectan el cumplimiento de otro. Revisamos la incoherencia entre dos instrumentos de política pública, el Programa de Acción Específico de Salud Materna y Perinatal (2013-2018) del Sistema Nacional de Salud y la certificación de los médicos especialistas de Ginecología y Obstetricia del Comité Normativo Nacional de Consejos de Especialidades Médicas (CONACEM) y los Consejos Médicos de Especialidades.

Sostenemos el argumento de que parte del problema en el uso discrecional de la práctica de la cesárea en México se puede apreciar ante la falta de coherencia existente entre instrumentos de política pública, en este caso, la asistencia a congresos médicos de ginecología y obstetricia.

La hipótesis que durante los días previos a los congresos la tasa de cesáreas aumenta, debido a que los médicos pueden programar anticipadamente nacimientos por cesárea para evitar que estos sucedan durante las fechas de los congresos y esto pueda comprometer su asistencia. Esto nos permite entender el uso discrecional de las cesáreas en México, en este caso provocado por la falta de coherencia entre políti- 
cas públicas. Analizamos la práctica en el uso de la cesárea tomando en cuenta la tasa de cesáreas antes, durante y después de los congresos. Los resultados señalan que los días previos al congreso registran un incremento en la tasa de cesáreas.

Estos resultados señalan la urgencia que tiene voltear a ver la coherencia entre políticas públicas en lo general, pero en lo particular, con la importancia de la práctica de las cesáreas. Actualmente la práctica de la cesárea es un tema que está tomando fuerza a nivel mundial porque se ha convertido en un problema de salud pública por el uso excesivo que se le ha dado en los últimos años.

El estudio ofrece implicaciones de política pública muy puntuales para el caso de las cesáreas, pero también para el análisis general de las políticas públicas. En lo particular, el estudio identifica un factor que incide en el uso discrecional de la cesárea como método de nacimiento; y deja entre ver que además de los factores de elección de este método asociados a la madre, factores no tan visibles relacionados con los médicos como la necesidad de asistir a congreso médicos de especialidad, tienen un papel importante en la elección del método de nacimiento en México.

El estudio pone sobre la mesa la necesidad de actualizar los planes de estudio de las instituciones educativas de formación de pregrado y posgrado para incorporar propuestas de procedimientos de nacimiento alternativos al uso de la cesárea. En México, el papel que desempeñan los médicos pasantes en servicio social y los médicos residentes es fundamental para los servicios de salud, la capacitación durante la formación académica para la atención del embarazo, parto y posparto, la identificación temprana de sus complicaciones y el conocimiento del tratamiento correspondiente son factores esenciales para la reducción de la práctica de la cesárea.

Lo anterior aunado a replantear diversas modalidades de acceso a capacitación especializada y certificación más allá de la asistencia física a congresos. Este elemento ha retomado fuerza recientemente a partir del uso de nuevas tecnologías para la educación y la divulgación de ciencia a distancia.

En lo general, el estudio identifica la necesidad de que los encargados de elaborar las políticas públicas (policy makers) durante el diseño sean conscientes de que los resultados de una política pública afectan los de otra; y que el éxito, más allá de la una buena lógica causal, depende del desempeño de otras políticas públicas.

Las propuestas de política pública sobre coherencia externa son dos. La primera tiene que ver con la necesidad de que durante el diseño de toda política pública se considere como parte del proceso el análisis de coherencia externa entre la política pública y otras políticas públicas relacionadas para una mejor comprensión de la sinergia entre ellas. La segunda propuesta muestra la necesidad de prestar atención al estudio de coherencia entre políticas públicas, con la finalidad de aumentar el conocimiento sobre este tema poco explorado aún y cuando su estudio y comprensión, podría implicar la solución a algunos problemas sobre la implementación y los resultados de las políticas públicas.

\section{ANEXO 1. ESPECIFICACIONES DE REGRESIÓN POR MÍNIMOS CUADRADOS ORDINARIOS}

\begin{tabular}{l|c}
\hline \multicolumn{1}{c}{ Variable } & $\begin{array}{c}\text { Todas variables } \mathbf{y} \\
\text { categorías }\end{array}$ \\
\hline \multirow{2}{*}{5 días } & Antes del congreso \\
\hline \multirow{2}{*}{4 días } & 0,000458 \\
\hline \multirow{2}{*}{3 días } & $(0,0763)$ \\
\cline { 2 - 2 } & $0,325^{* * *}$ \\
\hline \multirow{2}{*}{2 días } & $(0,0713)$ \\
\cline { 2 - 2 } & $0,189^{* * *}$ \\
\hline
\end{tabular}


GAPP. Nueva Época - N. 24, noviembre 2020 - ISSN: 1989-8991 - DOI: https://doi.org/10.24965/gapp.i24.10792 - [Págs. 24-43]

Los efectos de políticas públicas incoherentes. La relación entre los congresos médicos y el uso discrecional de la cesárea en México Ana Jeanette Rojas Cruz / Oliver David Meza Canales

\begin{tabular}{l|c}
\hline \multirow{2}{*}{1 día } & $0,193^{* * *}$ \\
\cline { 2 - 2 } & \multicolumn{2}{|c}{ Del congreso } \\
\hline \multirow{2}{*}{1 día } & $-0,580^{* * *}$ \\
\hline \multirow{2}{*}{2 día } & $(0,0712)$ \\
\hline \multirow{3}{*}{3 día } & $-0,737^{* * *}$ \\
\cline { 2 - 2 } & $(0,0712)$ \\
\hline \multirow{3}{*}{4 día } & $-0,0680$ \\
\hline \multirow{2}{*}{5 día } & $(0,0712)$ \\
\hline & $-0,171^{* *}$ \\
\hline
\end{tabular}

\begin{tabular}{|c|c|}
\hline \multicolumn{2}{|c|}{ Después del congreso } \\
\hline \multirow{2}{*}{1 día } & $-0,0444$ \\
\hline & $(0,0710)$ \\
\hline \multirow{2}{*}{2 día } & $0,142^{\star *}$ \\
\hline & $(0,0711)$ \\
\hline \multirow{2}{*}{3 día } & $-0,257^{* * *}$ \\
\hline & $(0,0711)$ \\
\hline \multirow{2}{*}{4 día } & $-0,0627$ \\
\hline & $(0,0711)$ \\
\hline \multirow{2}{*}{5 día } & $0,359^{* * *}$ \\
\hline & $(0,0761)$ \\
\hline \multirow{2}{*}{ Sede congreso } & $0,302^{* * *}$ \\
\hline & $(0,0223)$ \\
\hline \multicolumn{2}{|c|}{ Años } \\
\hline \multirow{2}{*}{2009} & $0,224^{* * *}$ \\
\hline & $(0,0147)$ \\
\hline \multirow{2}{*}{2010} & $0,276^{* * *}$ \\
\hline & $(0,0147)$ \\
\hline \multirow{2}{*}{2011} & $0,381^{* * *}$ \\
\hline & $(0,0147)$ \\
\hline \multirow{2}{*}{2012} & $0,445^{* * *}$ \\
\hline & $(0,0147)$ \\
\hline \multirow{2}{*}{2013} & $0,441^{* * *}$ \\
\hline & $(0,0147)$ \\
\hline
\end{tabular}


GAPP. Nueva Época - N. 24, noviembre 2020 - ISSN: 1989-8991 - DOI: https://doi.org/10.24965/gapp.i24.10792 - [Págs. 24-43]

Los efectos de políticas públicas incoherentes. La relación entre los congresos médicos y el uso discrecional de la cesárea en México Ana Jeanette Rojas Cruz / Oliver David Meza Canales

\begin{tabular}{|c|c|}
\hline \multirow{2}{*}{2014} & $0,387^{* * *}$ \\
\hline & $(0,0147)$ \\
\hline \multirow{2}{*}{2015} & $0,196^{* * *}$ \\
\hline & $(0,0147)$ \\
\hline \multicolumn{2}{|c|}{ Meses } \\
\hline \multirow{2}{*}{ Febrero } & $-0,0666^{\star \star *}$ \\
\hline & $(0,0183)$ \\
\hline \multirow{2}{*}{ Marzo } & $0,0658^{* \star *}$ \\
\hline & $(0,0179)$ \\
\hline \multirow{2}{*}{ Abril } & $-0,00736$ \\
\hline & $(0,0180)$ \\
\hline \multirow{2}{*}{ Mayo } & $0,0537^{\star * *}$ \\
\hline & $(0,0179)$ \\
\hline \multirow{2}{*}{ Junio } & $0,122^{\star \star *}$ \\
\hline & $(0,0180)$ \\
\hline \multirow{2}{*}{ Julio } & $0,332^{\star \star *}$ \\
\hline & $(0,0179)$ \\
\hline \multirow{2}{*}{ Agosto } & $0,602^{* * *}$ \\
\hline & $(0,0179)$ \\
\hline \multirow{2}{*}{ Septiembre } & $0,855^{\star * *}$ \\
\hline & $(0,0180)$ \\
\hline \multirow{2}{*}{ Octubre } & $0,601^{* \star *}$ \\
\hline & $(0,0186)$ \\
\hline \multirow{2}{*}{ Noviembre } & $0,284^{* * *}$ \\
\hline & $(0,0183)$ \\
\hline \multirow{2}{*}{ Diciembre } & $0,0335^{*}$ \\
\hline & $(0,0179)$ \\
\hline \multicolumn{2}{|c|}{ Días semana } \\
\hline \multirow{2}{*}{ Lunes } & $1,656^{* * *}$ \\
\hline & $(0,0139)$ \\
\hline \multirow{2}{*}{ Martes } & $1,980^{* \star *}$ \\
\hline & $(0,0139)$ \\
\hline \multirow{2}{*}{ Miércoles } & $1,947^{* \star *}$ \\
\hline & $(0,0139)$ \\
\hline \multirow{2}{*}{ Jueves } & $1,925^{* * *}$ \\
\hline & $(0,0139)$ \\
\hline
\end{tabular}


GAPP. Nueva Época - N. 24, noviembre 2020 - ISSN: 1989-8991 - DOI: https://doi.org/10.24965/gapp.i24.10792 - [Págs. 24-43]

Los efectos de políticas públicas incoherentes. La relación entre los congresos médicos y el uso discrecional de la cesárea en México Ana Jeanette Rojas Cruz / Oliver David Meza Canales

\begin{tabular}{l|c}
\hline \multirow{2}{*}{ Viernes } & $1,891^{* * *}$ \\
\cline { 2 - 2 } & $(0,0139)$ \\
\hline \multirow{2}{*}{ Sábado } & $0,810^{* * *}$ \\
\cline { 2 - 2 } & $(0,0139)$ \\
\hline
\end{tabular}

\section{Entidades federativas}

\begin{tabular}{|c|c|}
\hline \multirow{2}{*}{ Baja California } & $-1,620^{* * *}$ \\
\hline & $(0,0296)$ \\
\hline \multirow{2}{*}{ Baja California Sur } & $-0,729^{* * *}$ \\
\hline & $(0,0294)$ \\
\hline \multirow{2}{*}{ Campeche } & $-1,398^{* * *}$ \\
\hline & $(0,0294)$ \\
\hline \multirow{2}{*}{ Chiapas } & $-0,183^{* * *}$ \\
\hline & $(0,0294)$ \\
\hline \multirow{2}{*}{ Chihuahua } & $13,29^{* * *}$ \\
\hline & $(0,0295)$ \\
\hline \multirow{2}{*}{ Coahuila } & $-2,946^{* * *}$ \\
\hline & $(0,0294)$ \\
\hline \multirow{2}{*}{ Colima } & $-4,597^{* * *}$ \\
\hline & $(0,0294)$ \\
\hline \multirow{2}{*}{ Ciudad de México } & $-1,069^{* * *}$ \\
\hline & $(0,0295)$ \\
\hline \multirow{2}{*}{ Durango } & $-1,752^{* * *}$ \\
\hline & $(0,0294)$ \\
\hline \multirow{2}{*}{ Guanajuato } & $-0,361^{* * *}$ \\
\hline & $(0,0294)$ \\
\hline \multirow{2}{*}{ Guerrero } & $-2,278^{* * *}$ \\
\hline & $(0,0295)$ \\
\hline \multirow{2}{*}{ Hidalgo } & $-0,837^{* * *}$ \\
\hline & $(0,0294)$ \\
\hline \multirow{2}{*}{ Jalisco } & $-0,552^{* * *}$ \\
\hline & $(0,0295)$ \\
\hline \multirow{2}{*}{ Edo. México } & $-1,255^{\star \star *}$ \\
\hline & $(0,0294)$ \\
\hline \multirow{2}{*}{ Michoacán } & $-1,055^{* * *}$ \\
\hline & $(0,0294)$ \\
\hline \multirow{2}{*}{ Morelos } & $-0,992^{* * *}$ \\
\hline & $(0,0294)$ \\
\hline
\end{tabular}


GAPP. Nueva Época - N. 24, noviembre 2020 - ISSN: 1989-8991 - DOI: https://doi.org/10.24965/gapp.i24.10792 - [Págs. 24-43]

Los efectos de políticas públicas incoherentes. La relación entre los congresos médicos y el uso discrecional de la cesárea en México Ana Jeanette Rojas Cruz / Oliver David Meza Canales

\begin{tabular}{|c|c|}
\hline \multirow{2}{*}{ Nayarit } & $-2,715^{\star * \star}$ \\
\hline & $(0,0294)$ \\
\hline \multirow{2}{*}{ Nuevo León } & $-0,559^{* * *}$ \\
\hline & $(0,0295)$ \\
\hline \multirow{2}{*}{ Oaxaca } & $-1,754^{* * *}$ \\
\hline & $(0,0294)$ \\
\hline \multirow{2}{*}{ Puebla } & $-0,479^{* * *}$ \\
\hline & $(0,0294)$ \\
\hline \multirow{2}{*}{ Querétaro } & $-0,151^{* * *}$ \\
\hline & $(0,0294)$ \\
\hline \multirow{2}{*}{ Quintana Roo } & $-1,316^{\star \star *}$ \\
\hline & $(0,0295)$ \\
\hline \multirow{2}{*}{ San Luis Potosí } & $-2,503^{* * *}$ \\
\hline & $(0,0294)$ \\
\hline \multirow{2}{*}{ Sinaloa } & $-0,616^{\star * *}$ \\
\hline & $(0,0294)$ \\
\hline \multirow{2}{*}{ Sonora } & $-1,328^{* * *}$ \\
\hline & $(0,0294)$ \\
\hline \multirow{2}{*}{ Tabasco } & $-0,132^{* * *}$ \\
\hline & $(0,0294)$ \\
\hline \multirow{2}{*}{ Tamaulipas } & $-0,486^{* * *}$ \\
\hline & $(0,0294)$ \\
\hline \multirow{2}{*}{ Tlaxcala } & $-0,239^{* * *}$ \\
\hline & $(0,0294)$ \\
\hline \multirow{2}{*}{ Veracruz } & $-1,981^{* * *}$ \\
\hline & $(0,0295)$ \\
\hline \multirow{2}{*}{ Yucatán } & $-0,599^{* \star *}$ \\
\hline & $(0,0294)$ \\
\hline \multirow{2}{*}{ Zacatecas } & $-2,281^{\text {*** }}$ \\
\hline & $(0,0294)$ \\
\hline \multirow{2}{*}{ Constante } & $3,611^{* * *}$ \\
\hline & $(0,0275)$ \\
\hline $\mathrm{N}$ & 93,495 \\
\hline R-cuadrada & 0,864 \\
\hline
\end{tabular}

\section{REFERENCIAS BIBLIOGRÁFICAS}

AGUILAR, L. F. (2009): "Marco para el análisis de las políticas públicas", en MARIÑEZ, F. y GARZA, V. (coords.): Política pública y democracia en América Latina: Del análisis a la implementación, págs. 11-33. México: Miguel Ángel Porrúa.

BELTRÁN, A. P.; YE, J.; MOLLER, A.-B.; ZHANG, J.; GÜLMEZOGLU, A. M.; TORLONI, M. ${ }^{a}$ R. (2016): "The Increasing Trend in Caesarean Section Rates: Global, Regional and National Estimates: 1990-2014", en PLoS ONE, vol. 11, núm. 2, e0148343. DOI: https://doi.org/10.1371/journal.pone.0148343. 
GAPP. Nueva Época - N. 24, noviembre 2020 - ISSN: 1989-8991 - DOI: https://doi.org/10.24965/gapp.i24.10792 - [Págs. 24-43]

Los efectos de políticas públicas incoherentes. La relación entre los congresos médicos y el uso discrecional de la cesárea en México

Ana Jeanette Rojas Cruz / Oliver David Meza Canales

BERNAL-GARCÍA C.; NAHÍN-ESCOBEDO, C. C. (2018): "Cesárea: situación actual y factores asociados en México", en Salud Quintana Roo, vol. 11, núm. 40, págs. 28-33. URL: https://www.medigraphic.com/cgi-bin/new/resumen. cgi?IDARTICULO=92469.

BLOUIN, C. (2007): "Trade Policy and Health: From Conflicting Interests to Policy Coherence", en Bulletin of the World Health Organization, vol. 85, núm. 3, págs. 169-173. DOI: https://doi.org/10.2471/blt.06.037413.

BURNS, L. R.; GELLER, S. E.; WHOLEY, D. R. (1995): "The effect of physician factors on the caesarean decision", en Medical Care, vol. 33, núm. 4, págs. 365-382. https://doi.org/10.1097/00005650-199504000-00004.

CEJUDO, G. M.; MICHEL, C. L. (2017): "Addressing fragmented government action: coordination, coherence, and integration", en Policy Sciences, vol. 50, págs. 745-767. DOI: https://doi.org/10.1007/s11077-017-9281-5.

CEJUDO, G. M.; MICHEL, C. L. (2016): “Coherencia y Políticas Públicas. Metas, instrumentos y poblaciones objetivo”, en Gestión y Política Pública, vol. 25, núm. 1, págs. 3-31. DOI: http://dx.doi.org/10.29265/gypp.v25i1.149.

CEJUDO, G. M.; MICHEL, C. L. (2015): "Resolviendo problemas sociales complejos mediante la integración de políticas. El caso de la Cruzada Nacional contra el Hambre en México", en Revista del CLAD Reforma y Democracia, núm. 63, págs. 33-64. URL: http://old.clad.org/portal/publicaciones-del-clad/revista-clad-reformademocracia/articulos/063-octubre-2015/Cejudo.pdf.

CHRISTENSEN, T.; LÆEGREID, P. (2008): "The Challenge of Coordination in Central Government Organizations: the Norwegian Case", en Public Organization Review, vol. 8, núm. 2, págs. 97-116. DOI: https://doi.org/10.1007/ s11115-008-0058-3.

CROWLEY, K.; HEAD, B. W. (2017): “The enduring challenge of «wicked problems»: revisiting Rittel and Webber”, en Policy Sciences, vol. 50, núm. 4, págs. 539-547. DOI: https://doi.org/10.1007/s11077-017-9302-4.

COMITÉ NORMATIVO NACIONAL DE CONSEJOS DE ESPECIALIDADES MÉDICAS A. C. (CONACEM) (2017): Reglamento de Idoneidad. Revisado en septiembre 2017. URL: https://conacem.org.mx/index.php/marco-juridico/ reglamento.

CONSEJO MEXICANO DE GINECOLOGÍA Y OBSTETRICIA A. C. (CMGO) (2017): Estatutos de la Asociación. Revisado en septiembre 2017. URL: https://cmgo.org.mx/assets/estatutos_cmgo_2020.pdf.

CONSEJO MEXICANO DE GINECOLOGÍA Y OBSTETRICIA A. C. (CMGO) (2017): Examen de certificación. Revisado en septiembre 2017. URL: https://www.cmgo.org.mx/certificacion.html.

CONSEJO MEXICANO DE GINECOLOGÍA Y OBSTETRICIA A. C. (CMGO) (2017): Cedula de Vigencia de Certificación. Revisado en septiembre 2017. URL: https://cmgo.org.mx/assets/cedula_vigencia_ certificacion_2019.pdf.

ESTADOS UNIDOOS MEXICANOS (2015): "Acuerdo por el que se emiten los Lineamientos a los que se sujetarán el Comité Normativo Nacional de Consejos de Especialidades Médicas y los Consejos de Especialidades Médicas a los que se refiere el art. 81 de la Ley General de Salud", en Diario Oficial de la Federación, 25 de marzo de 2015. Revisado en enero 2018. URL: $h t t p s: / / w w w . d o f . g o b . m x /$ nota_detalle.php?codigo=5386686\&fecha=25/03/2015.

DIRECCIÓN GENERAL DE INFORMACIÓN EN SALUD DE MÉXICO (2017): Datos Abiertos-Nacimientos. Revisado en julio 2017. URL: http://www.dgis.salud.gob.mx/contenidos/basesdedatos/da_nacimientos_gobmx. html.

ELIZALDE GONZÁLEZ, J. J. (2017): “Congresos médicos. La importancia de participar”, en Medicina Crítica, vol. 31, núm. 6, págs. 318-319. URL: https://www.medigraphic.com/pdfs/medcri/ti-2017/ti176a.pdf.

FEDERACIÓN MEXICANA DE COLEGIOS DE OBSTETRICIA Y GINECOLOGÍA A. C. (FEMECOG) (2017): Inicio. Revisado en septiembre 2017. URL: https://femecog.org.mx/.

GONZÁLEZ-PÉREZ, G. J.; GUADALUPE VEGA, M.a; CABRERA, C. (2011): "Factores socio epidemiológicos y de la organización de los servicios de salud asociados a la operación cesárea”, en GONZÁLEZ-PÉREZ, G. J.; GUADALUPE VEGA, M.a; CABRERA, C.: Cesáreas en México: aspectos sociales, económicos y epidemiológicos. México: Universidad de Guadalajara.

HEAD, B. W.; ALFORD, J. (2013): "Wicked Problems: Implications for Public Policy and Management", en Administration \& Society, vol. 47, núm. 6, págs. 711-739. DOI: https://doi.org/10.1177/0095399713481601.

HOWLETT, M.; RAYNER, J. (2007): "Design Principles for Policy Mixes: Cohesion and Coherence in «New Governance Arrangements»”, en Policy and Society, vol. 26, núm. 4, págs. 1-18. https://doi.org/10.1016/s14494035(07)70118-2.

HURST, M. y SUMMEY, P. (1984): "Childbirth and social class: The case cesarean delivery”, en Social Science and Medicine, vol. 18, núm. 8, págs. 621-631. https://doi.org/10.1016/0277-9536(84)90290-9.

INSTITUTO NACIONAL DE GEOGRAFÍA Y ESTADÍSTICA (INEGI) (2018): Comunicado de Prensa núm. 244/19 de la Encuesta Nacional de la Dinámica Demográfica (ENADID) 2018. Revisado en mayo 2019. URL: https://www. inegi.org.mx/contenidos/saladeprensa/boletines/2019/EstSociodemo/ENADID2018.pdf.

MUÑOZ-ENCISO J. M.; ROSALES-AUJANG, E.; DOMÍNGUEZ-PONCE, G.; SERRANO-DÍAZ C. (2011): "Operación cesárea: ¿Indicación justificante o preocupación justificada?”, en Ginecología y Obstetricia de México, vol. 79, núm. 2, págs. 67-74. URL: https://www.medigraphic.com/cgi-bin/new/resumen.cgi?IDARTICULO=28187.

JUÁREZ, J. (2017): "Una epidemia de cesáreas innecesarias en México", en The New York Times. Consultado en septiembre 2017. URL: https://www.nytimes.com/es/2017/08/28/una-epidemia-de-cesareas-innecesarias-enmexico/?smid=tw-share-es. 
GAPP. Nueva Época - N. 24, noviembre 2020 - ISSN: 1989-8991 - DOI: https://doi.org/10.24965/gapp.i24.10792 - [Págs. 24-43]

Los efectos de políticas públicas incoherentes. La relación entre los congresos médicos y el uso discrecional de la cesárea en México Ana Jeanette Rojas Cruz / Oliver David Meza Canales

MAY, P. J.; SAPOTICHNE, J.; WORKMAN, S. (2006): "Policy Coherence and Policy Domains", en The Policy Studies Journal, vol. 34, núm. 3, págs. 381-403. DOI: https://doi.org/10.1111/j.1541-0072.2006.00178.x.

MEZA, O. (2018): "Del plato a la boca se cae la sopa: evaluación de la consistencia en las intervenciones públicas subnacionales”, en BALLEZCÁ, M. (ed.): Evaluación de Políticas Subnacionales en México: Evalúa Jalisco, págs. 287-309. Guadalajara: Gobierno de Jalisco y Colegio de Jalisco.

MEZA, O.; GUERRA E.; CORONA, G. (2018): "Las Políticas de Drogas y el Estado: Una aproximación para una política de mitigación de riesgos”, en Boletim de Análise Político-Institucional Diciembre, núm. 18, págs. 19-29.

NEWMAN, J.; HEAD, B. W. (2017): "Wicked tendencies in policy problems: Rethinking the distinction between social and technical problems", en Policy and Society, vol. 36, núm. 3, págs. 414-429. DOI: https://doi.org/10.1080/14494 035.2017.1361635.

ORGANIZACIÓN MUNDIAL DE LA SALUD Y HUMAN REPRODUCTIVE PROGRAMME (2015): Declaración de la OMS sobre la tasa de cesáreas. Resumen ejecutivo. Revisado en septiembre 2017. URL: http://www.who.int/ reproductivehealth/publications/maternal_perinatal_health/cs-statement/es/.

PETERS, G. B. (1998): "Managing Horizontal Government: The Politics of Coordination", en Public Administration, vol. 76, núm. 1, págs. 295-311. DOI: https://doi.org/10.1111/1467-9299.00102.

PETERS, G. B. (2004): The Search for Coordination and Coherence in Public Policy: Return to the Center? Pittsburgh: University of Pittsburgh.

POLLIT, C. (2003): "Joined up government: a survey”, en Political Studies Review, vol. 1, núm. 1, págs. 34-49. https:// doi.org/10.1111/1478-9299.00004.

RITTEL, H. W. J.; WEBBER. M. M. (1973): "Dilemmas in a general theory of planning", en Policy Sciences, vol. 4, núm. 1.973, págs. 155-169. https://doi.org/10.1007/BF01405730.

SANDALL, J.; TRIBE, R.; AVERY, L.; MOLA, G.; VISSER, G.; HOMER, C.; GIBBONS, D.; KELLY, N.; POWELL, H.; KIDANTO, H.; TAYLOR, P.; TEMMERMAN, M. (2018): "Short-term and long term effects of caesarean section on the health of women and children”, en The Lancet, vol. 392, núm. 10.155, págs. 1.349-1.357. https://doi. org/10.1016/S0140-6736(18)31930-5.

SECRETARÍA DE SALUD (SSA) (2002): Cesárea segura. Lineamiento técnico. Revisado en septiembre 2017. URL: http://www.salud.gob.mx/unidades/cdi/documentos/DOCSAL7101.pdf.

STEAD, D. (2008): "Institutional aspects of integrating transport, environment and healt policies", en Transport Policy, vol. 15, núm. 3, págs. 139-148. https://doi.org/10.1016/j.tranpol.2007.12.001.

TUSSING, A. D.; WOJTOWICZ, M. A. (1993): "The effect of physician characteristics on clinical behavior: Cesarean section in New York State", en Social Science and Medicine, vol. 37, núm. 10, págs. 1.251-1.260. https://doi. org/10.1016/0277-9536(93)90336-3.

VALLEJOS-PARÁS A.; ESPINO-Y-SOSA, S.; RUIZ-SÁNCHEZ, J.; DURÁN-ARENAS, L. (2011): "Tendencias y factores asociados a cesáreas en México: validación de un instrumento", en Perinatología y Reproducción Humana, vol. 25, núm. 4, págs. 212-218.

WOLPERT, E. (2017): "La certificación médica”, en Memoria simposio CONAMED. La Constitución y la Salud, vol. 5, núm. 5 , págs. 1-14.

WOOLDRIDGE, J. M. (2009): “Análisis de regresión múltiple”, en WOOLDRIDGE, J. M. (coord.): Introducción a la econometría. Un enfoque moderno, págs. 73-112. México: Cengage Learning. 